\title{
A Superficial Tale: Semiconductor Nanowires as a Remarkable Platform for Nanoelectronics and Sensing
}

\author{
Harry E. Ruda, \\ University of Toronto, Canada \\ harry.ruda@utoronto.ca
}

\begin{abstract}
The first foray into semiconductor micron-scale 'whiskers' came from work by Wagner and Ellis in 1964, only to applied in the late 1990's to realise nanowires with diameters of tens of nanometers. With the possibility of strong confinement in two dimensions, these structures present ideal vehicles for 1d physics and devices. However, surface related phenomena can provide a curse or opportunity in this quest - the latter is the focus of this presentation. Here, I focus on the opportunities in a few areas including ballistic conductance, random telegraph noise, and scattering from individual surface charges. Harnessing these phenomena can enable a host of new opportunities including making inroads in the quest to tame the elusive Majorana Fermion, in ultra-sensitive elevated temperature single charge electrometry and in single molecule level sensing.
\end{abstract}

